\title{
Optimal Lower Generalized Logarithmic Mean Bound for the Seiffert Mean
}

\author{
Ying-Qing Song, ${ }^{1}$ Wei-Mao Qian, ${ }^{2}$ Yun-Liang Jiang, ${ }^{3}$ and Yu-Ming Chu ${ }^{1}$ \\ ${ }^{1}$ School of Mathematics and Computation Sciences, Hunan City University, Yiyang, Hunan 413000, China \\ ${ }^{2}$ School of Distance Education, Huzhou Broadcast and TV University, Huzhou, Zhejiang 313000, China \\ ${ }^{3}$ School of Information \& Engineering, Huzhou Teachers College, Huzhou, Zhejiang 313000, China
}

Correspondence should be addressed to Yun-Liang Jiang; jylsy@hutc.zj.cn

Received 23 December 2012; Accepted 26 February 2013

Academic Editor: Francisco J. Marcellán

Copyright (c) 2013 Ying-Qing Song et al. This is an open access article distributed under the Creative Commons Attribution License, which permits unrestricted use, distribution, and reproduction in any medium, provided the original work is properly cited.

We present the greatest value $p$ such that the inequality $P(a, b)>L_{p}(a, b)$ holds for all $a, b>0$ with $a \neq b$, where $P(a, b)$ and $L_{p}(a, b)$ denote the Seiffert and $p$ th generalized logarithmic means of $a$ and $b$, respectively.

\section{Introduction}

For $p \in \mathbb{R}$, the $p$ th generalized logarithmic mean $L_{p}:$ $(0, \infty)^{2} \rightarrow(0, \infty)$ is defined by

$$
L_{p}(a, b)= \begin{cases}{\left[\frac{b^{p+1}-a^{p+1}}{(p+1)(b-a)}\right]^{1 / p},} & a \neq b, p \neq 0,-1, \\ \frac{1}{e}\left(\frac{b^{b}}{a^{a}}\right)^{1 /(b-a)}, & a \neq b, p=0, \\ \frac{b-a}{\log b-\log a}, & a \neq b, p=-1, \\ a, & a=b,\end{cases}
$$

and the Seiffert mean $P:(0, \infty)^{2} \rightarrow(0, \infty)[1]$ is defined by

$$
P(a, b)= \begin{cases}\frac{a-b}{4 \arctan (\sqrt{a / b})-\pi}, & a \neq b, \\ a, & a=b .\end{cases}
$$

It is well known that the generalized logarithmic mean $L_{p}(a, b)$ is continuous and strictly increasing with respect to $p \in \mathbb{R}$ for fixed $a, b>0$ with $a \neq b$. The special cases of the generalized logarithmic mean are, for example, $G(a, b)=$ $\sqrt{a b}=L_{-2}(a, b)$ is the geometric mean, $L(a, b)=(b-a) /$ $(\log b-\log a)=L_{-1}(a, b)$ is the logarithmic mean, $I(a, b)=$
$1 / e\left(b^{b} / a^{a}\right)^{1 /(b-a)}=L_{0}(a, b)$ is the identric mean, and $A(a, b)=(a+b) / 2=L_{1}(a, b)$ is the arithmetic mean. The Seiffert mean $P(a, b)$ can be rewritten as (see [2, equation (2.4)])

$$
P(a, b)= \begin{cases}\frac{a-b}{2 \arcsin [(a-b) /(a+b)]}, & a \neq b, \\ a, & a=b .\end{cases}
$$

Recently, the bivariate means have been the subject of intensive research. In particular, many remarkable inequalities and properties for the generalized logarithmic and the Seiffert means can be found in the literature [3-13].

In $[1,11]$, Seiffert proved that the inequalities

$$
\begin{gathered}
L_{-1}(a, b)<P(a, b)<L_{0}(a, b)=I(a, b) \\
\frac{2}{\pi} A(a, b)<P(a, b)<A(a, b) \\
P(a, b)>\frac{G(a, b) A(a, b)}{L(a, b)} \\
P(a, b)>\frac{3 A(a, b) G(a, b)}{A(a, b)+2 G(a, b)}
\end{gathered}
$$

hold for all $a, b>0$ with $a \neq b$. 
Sándor [14] presented the bounds for the Seiffert mean $P(a, b)$ in terms of the arithmetic mean $A(a, b)$ and geometric mean $G(a, b)$ as follows:

$$
\frac{1}{2} A(a, b)+\frac{1}{2} G(a, b)<P(a, b)<\frac{2}{3} A(a, b)+\frac{1}{3} G(a, b)
$$

for all $a, b>0$ with $a \neq b$.

Hästö [15] proved that the double inequality $M_{\alpha}(a, b)<$ $P(a, b)<M_{\beta}(a, b)$ holds for all $a, b>0$ with $a \neq b$ if and only if $\alpha \leq \log 2 / \log \pi$ and $\beta \geq 2 / 3$, where $M_{p}(a, b)=\left(\left(a^{p}+\right.\right.$ $\left.\left.b^{p}\right) / 2\right)^{1 / p}$ is the $p$ th power mean of $a$ and $b$.

In [16], the authors found the greatest value $\alpha$ and least value $\beta$ such that the double inequality $\alpha C(a, b)+(1-$ $\alpha) G(a, b)<P(a, b)<\beta C(a, b)+(1-\beta) G(a, b)$ holds for all $a, b>0$ with $a \neq b$, where $C(a, b)=\left(a^{2}+b^{2}\right) /(a+b)$ is the contraharmonic mean of $a$ and $b$.

Motivated by the first inequality in (4), Gao [17] gave the best possible constants $\lambda$ and $\mu$ such that the double inequality $\lambda I(a, b)<P(a, b)<\mu I(a, b)$ holds for all $a, b>0$ with $a \neq b$.

In [18], the author solved the following open problem proposed by Long and Chu [19]: what is the smallest $p$ (largest $q$ ) such that the inequality $\alpha A(a, b)+(1-\alpha) G(a, b)<L_{p}(a, b)(>$ $\left.L_{q}(a, b)\right)$ holds for $\alpha \in(0,1 / 2)(\alpha \in(1 / 2,1))$ and all $a, b>0$ with $a \neq b$ ?

Chu et al. [20] proved that the double inequality $L_{p}(a, b)<T(a, b)<L_{q}(a, b)$ holds for all $a, b>0$ with $a \neq b$ if and only if $p \leq 3$ and $q \geq q_{0}=3.152 \ldots$, where $T(a, b)=$ $(a-b) /[2 \arctan ((a-b) /(a+b))]$ is the second Seiffert mean of $a$ and $b$, and $p_{0}=3.152 \ldots$ is the unique solution of the equation $(x+1)^{-1 / x}=2 / \pi$.

In [21], the authors answered the question: what are the greatest value $p_{1}=p_{1}(q)$ and the least value $p_{2}=p_{2}(q)$ such that the double inequality $L_{p_{1}}(a, b)<\left[L\left(a^{q}, b^{q}\right)\right]^{1 / q}<$ $L_{p_{2}}(a, b)$ holds for any $q>0$ with $q \neq 1$ and all $a, b>0$ with $a \neq b$ ?

Motivated by the first inequality in (4), it is natural to ask what are the best possible generalized logarithmic mean bounds for the Seiffert mean $P(a, b)$ ? It is the aim of this paper to answer this question.

\section{Preliminaries}

In order to prove our main results, we need two lemmas, which we present in this section.

Lemma 1. Let the function $E:(-1,+\infty) \rightarrow \mathbb{R}_{+}$be defined with

$$
\begin{gathered}
(x+1)^{-1 / x}, \quad x \in(-1,0) \cup(0,+\infty), \\
\frac{1}{e}, \quad x=0 .
\end{gathered}
$$

Then, $E$ is a continuous and strictly increasing function.
Proof. From (6), we clearly see that

$$
\begin{gathered}
\lim _{x \rightarrow 0} E(x)=\frac{1}{e}=E(0), \\
\lim _{x \rightarrow-1^{+}} E(x)=0, \\
\lim _{x \rightarrow+\infty} E(x)=1 .
\end{gathered}
$$

If $x \in(-1,0) \cup(0,+\infty)$, then simple computation yields

$$
E^{\prime}(x)=\frac{(1+x) \log (1+x)-x}{x^{2}(1+x)} E(x)
$$

If we define

$$
F(x)=(1+x) \log (1+x)-x,
$$

then

$$
F^{\prime}(x)=\log (1+x) .
$$

Equation (11) implies that

$$
\begin{aligned}
& F^{\prime}(x)<0, \quad x \in(-1,0), \\
& F^{\prime}(x)>0, \quad x \in(0,+\infty) .
\end{aligned}
$$

Equations (10) and (12) lead to

$$
F(x)>\lim _{x \longrightarrow 0} F(x)=0
$$

for $x \in(-1,0) \cup(0,+\infty)$.

From (9) and (10) together with (13), we clearly see that

$$
E^{\prime}(x)>0
$$

for $x \in(-1,0) \cup(0,+\infty)$.

Therefore, the continuity of $E$ follows from (6) and (7), and the strict monotonicity of $E$ follows from (8), (14) and the continuity of $E$.

Remark 2. From Lemma 1, we clearly see that for any fixed $\lambda \in(0,1)$, there exists a unique $x \in(-1, \infty)$ such $E(x)=\lambda$. In particular, for $\lambda=1 / \pi$, making use of Mathematica software, we get

$$
\begin{gathered}
E(-0.241)-\frac{1}{\pi}=0.000166 \cdots>0, \\
E(-0.242)-\frac{1}{\pi}=-0.000062 \cdots<0 .
\end{gathered}
$$

Therefore, the unique solution of the equation $E(x)=1 / \pi$ belongs to the interval $(-0.242,-0.241)$.

Lemma 3. Let $p \in(-0.242,-0.241)$ and let $g:[1,+\infty) \rightarrow$ $\mathbb{R}$ be defined with $g(t)=p t^{2 p+3}+2(p-1) t^{2 p+2}-(3 p+2) t^{2 p+1}-$ $p(2 p+1) t^{p+3}+\left(2 p^{2}+p+4\right) t^{p+2}+\left(2 p^{2}+p+4\right) t^{p+1}-p(2 p+$ 1) $t^{p}-(3 p+2) t^{2}+2(p-1) t+p$. Then, there exists $\lambda \in(1,+\infty)$ such that $g(t)<0$ for $t \in(1, \lambda)$ and $g(t)>0$ for $t \in(\lambda,+\infty)$. 
Proof. Simple computations lead to

$$
\begin{aligned}
& g(1)=0, \\
& \lim _{t \rightarrow+\infty} g(t)=+\infty \text {, } \\
& g^{\prime}(t)=p(2 p+3) t^{2 p+2}+4(p-1)(p+1) t^{2 p+1} \\
& -(2 p+1)(3 p+2) t^{2 p}-p(p+3)(2 p+1) t^{p+2} \\
& +(p+2)\left(2 p^{2}+p+4\right) t^{p+1}+(p+1) \\
& \times\left(2 p^{2}+p+4\right) t^{p}-p^{2}(2 p+1) t^{p-1} \\
& -2(3 p+2) t+2(p-1) \text {, } \\
& g^{\prime}(1)=0 \text {, } \\
& \lim _{t \longrightarrow+\infty} g^{\prime}(t)=+\infty \\
& g^{\prime \prime}(t)=2 p(p+1)(2 p+3) t^{2 p+1} \\
& +4(p-1)(p+1)(2 p+1) t^{2 p}-2 p \\
& \times(2 p+1)(3 p+2) t^{2 p-1} \\
& -p(p+2)(p+3)(2 p+1) t^{p+1} \\
& +(p+1)(p+2)\left(2 p^{2}+p+4\right) t^{p} \\
& +p(p+1)\left(2 p^{2}+p+4\right) t^{p-1} \\
& -p^{2}(p-1)(2 p+1) t^{p-2}-2(3 p+2), \\
& g^{\prime \prime}(1)=0 \text {, } \\
& \lim _{t \rightarrow+\infty} g^{\prime \prime}(t)=+\infty \text {. }
\end{aligned}
$$

Let $h(t)=t^{3-p} g^{\prime \prime \prime}(t)$. Further computations lead to

$$
\begin{aligned}
h(t)= & 2 p(p+1)(2 p+1)(2 p+3) t^{p+3} \\
& +8 p(p-1)(p+1)(2 p+1) t^{p+2} \\
& -2 p(2 p-1)(2 p+1)(3 p+2) t^{p+1} \\
& -p(p+1)(p+2)(p+3) \\
& \times(2 p+1) t^{3}+p(p+1)(p+2) \\
& \times\left(2 p^{2}+p+4\right) t^{2}+p(p-1) \\
& \times(p+1)\left(2 p^{2}+p+4\right) t \\
& -p^{2}(p-2)(p-1)(2 p+1), \\
& h(1)=0,
\end{aligned}
$$

$$
\lim _{t \rightarrow+\infty} h(t)=+\infty
$$

$$
\begin{aligned}
h^{\prime}(t)= & 2 p(p+1)(p+3)(2 p+1)(2 p+3) t^{p+2} \\
& +8 p(p-1)(p+1)(p+2)(2 p+1) t^{p+1} \\
& -2 p(p+1)(2 p-1)(2 p+1)(3 p+2) t^{p} \\
& -3 p(p+1)(p+2)(p+3)(2 p+1) t^{2} \\
& +2 p(p+1)(p+2)\left(2 p^{2}+p+4\right) t+p \\
& \times(p-1)(p+1)\left(2 p^{2}+p+4\right), \\
& h^{\prime}(1)=24 p^{3}(p+1)<0,
\end{aligned}
$$

$h^{\prime \prime}(t)=2 p(p+1)(p+2)(p+3)(2 p+1)(2 p+3) t^{p+1}$

$$
+8 p(p-1)(p+1)^{2}(p+2)(2 p+1) t^{p}
$$$$
-2 p^{2}(p+1)(2 p-1)(2 p+1)(3 p+2) t^{p-1}
$$$$
-6 p(p+1)(p+2)(p+3)(2 p+1) t
$$$$
+2 p(p+1)(p+2)\left(2 p^{2}+p+4\right)
$$

$$
h^{\prime \prime}(1)=p^{3}\left(72 p^{2}+156 p+84\right)<0,
$$

$$
\lim _{t \rightarrow+\infty} h^{\prime \prime}(t)=+\infty
$$

$$
\begin{aligned}
h^{\prime \prime \prime}(t)= & 2 p(p+1)^{2}(p+2)(p+3)(2 p+1) \\
& \times(2 p+3) t^{p}+8 p^{2}(p-1)(p+1)^{2} \\
& \times(p+2)(2 p+1) t^{p-1}-2 p^{2}(p-1) \\
& \times(p+1)(2 p-1)(2 p+1) \\
& \times(3 p+2) t^{p-2}-6 p(p+1) \\
& \times(p+2)(p+3)(2 p+1),
\end{aligned}
$$$$
h^{\prime \prime \prime}(1)=p^{2}\left(112 p^{4}+312 p^{3}+352 p^{2}+192 p+40\right)>0 \text {, }
$$

$$
\begin{aligned}
h^{(4)}(t)= & 2 p^{2}(p+1)^{2}(p+2)(p+3) \\
& \times(2 p+1)(2 p+3) t^{p-1} \\
& +8 p^{2}(p-1)^{2}(p+1)^{2}(p+2)(2 p+1) t^{p-2} \\
& +2 p^{2}(1-p)(2-p)(1-2 p) \\
& \times(p+1)(2 p+1)(3 p+2) t^{p-3} \\
> & 0 .
\end{aligned}
$$


Inequalities (34) and (35) imply that

$$
h^{\prime \prime \prime}(t)>0
$$

for $t \in(1, \infty)$.

From (31) and (32) together with (36), we clearly see that there exists $t_{0} \in(1, \infty)$ such that $h^{\prime \prime}(t)<0$ for $t \in\left(1, t_{0}\right)$ and $h^{\prime \prime}(t)>0$ for $t \in\left(t_{0}, \infty\right)$. Hence, $h^{\prime}$ is strictly decreasing on $\left(1, t_{0}\right)$ and strictly increasing on $\left(t_{0}, \infty\right)$.

It follows from (28) and (29) together with the monotonicity of $h^{\prime}$ that there exists $t_{1} \in(1, \infty)$ such that $h$ is strictly decreasing on $\left(1, t_{1}\right)$ and strictly increasing on $\left(t_{1}, \infty\right)$.

Equations (25)-(26) and the monotonicity of $h$ lead to the conclusion that there exists $t_{2} \in(1, \infty)$ such that $h(t)<0$ for $t \in\left(1, t_{2}\right)$ and $h(t)>0$ for $t \in\left(t_{2}, \infty\right)$. Therefore, $g^{\prime \prime}$ is strictly decreasing on $\left(1, t_{2}\right)$ and strictly increasing on $\left(t_{2}, \infty\right)$.

From (22) and (23) together with the monotonicity of $g^{\prime \prime}$, we clearly see that there exists $t_{3} \in(1, \infty)$ such that $g^{\prime}$ is strictly decreasing on $\left(1, t_{3}\right)$ and strictly increasing on $\left(t_{3}, \infty\right)$.

Equations (19)-(20) and the monotonicity of $g^{\prime}$ imply that there exists $t_{4} \in(1, \infty)$ such that $g$ is strictly decreasing on $\left(1, t_{4}\right)$ and strictly increasing on $\left(t_{4}, \infty\right)$.

Therefore, Lemma 3 follows from (16) and (17) together with the monotonicity of $g$.

\section{Main Results}

Theorem 4. Let $E$ be as in Lemma 1 and let $p$ be the unique solution of the equation $E(x)=1 / \pi$. Then, for all $a, b>0$, $a \neq b$, the inequality

$$
L_{p}(a, b)<P(a, b)
$$

holds, and $L_{p}(a, b)$ is the best possible lower generalized logarithmic mean bound for the Seiffert mean $P(a, b)$.

Proof. From Remark 2, it follows that

$$
p \in(-0.242,-0.241) \text {. }
$$

We first prove that the inequality (37) holds. Without loss of generality, we assume that $a>b$. If $t=a / b>1$, then, from (1) and (2), we have

$$
\begin{aligned}
\log L_{p}(a, b)-\log P(a, b) \\
=\frac{1}{p} \log \frac{t^{p+1}-1}{(p+1)(t-1)} \\
\quad+\log (4 \arctan \sqrt{t}-\pi)-\log (t-1),
\end{aligned}
$$

If

$$
\begin{aligned}
f(t)= & \frac{1}{p} \log \frac{t^{p+1}-1}{(p+1)(t-1)} \\
& +\log (4 \arctan \sqrt{t}-\pi)-\log (t-1),
\end{aligned}
$$

then simple computations lead to

$$
\lim _{t \rightarrow 1} f(t)=\lim _{t \longrightarrow+\infty} f(t)=0,
$$

$$
f^{\prime}(t)=\frac{(p+1)\left(t^{p}-1\right)}{p\left(t^{p+1}-1\right)(t-1)(4 \arctan \sqrt{t}-\pi)} f_{1}(t)
$$

where

$$
\begin{gathered}
f_{1}(t)=-4 \arctan \sqrt{t}+\pi+\frac{2 p\left(t^{p+1}-1\right)(t-1)}{(p+1) \sqrt{t}(t+1)\left(t^{p}-1\right)}, \\
\lim _{t \rightarrow 1} f_{1}(t)=0, \\
\lim _{t \rightarrow+\infty} f_{1}(t)=+\infty, \\
f_{1}^{\prime}(t)=\frac{f_{2}(t)}{(1+p)(t+1)^{2}\left(t^{p}-1\right)^{2} t^{3 / 2}},
\end{gathered}
$$

where

$$
\begin{aligned}
f_{2}(t)= & p t^{2 p+3}+2(p-1) t^{2 p+2}-(3 p+2) t^{2 p+1} \\
& -p(2 p+1) t^{p+3}+\left(2 p^{2}+p+4\right) t^{p+2} \\
& +\left(2 p^{2}+p+4\right) t^{p+1}-p(2 p+1) t^{p} \\
& -(3 p+2) t^{2}+2(p-1) t+p .
\end{aligned}
$$

From (38) and (44)-(45) together with Lemma 3, we clearly see that there exists $\lambda \in(1,+\infty)$ such that $f_{1}$ is strictly decreasing on $(1, \lambda)$ and strictly increasing on $(\lambda, \infty)$. Then, (38) and (42)-(43) together with the monotonicity of $f_{1}$ imply that there exists $\mu \in(1,+\infty)$ such that $f$ is strictly decreasing on $(1, \mu)$ and strictly increasing on $(\mu,+\infty)$.

Therefore, $L_{p}(a, b)<P(a, b)$ follows from (39)-(41) and the monotonicity of $f$.

Next, we prove that $L_{p}(a, b)$ is the best possible lower generalized logarithmic mean bound for the Seiffert mean $P(a, b)$.

For any $0<\varepsilon<-p$, from (1) and (2), we get

$$
\lim _{t \rightarrow+\infty} \frac{L_{p+\varepsilon}(t, 1)}{P(t, 1)}=\pi(p+1+\varepsilon)^{-1 /(p+\varepsilon)} .
$$

Lemma 1 and (46) lead to

$$
\lim _{t \rightarrow+\infty} \frac{L_{p+\varepsilon}(t, 1)}{P(t, 1)}>1 .
$$

Inequality (47) implies that, for $0<\varepsilon<-p$, there exists $T=T(\varepsilon)>1$ such that $L_{p+\varepsilon}(t, 1)>P(t, 1)$ for $t \in(T, \infty)$.

Theorem 5. $L_{0}(a, b)$ is the best possible upper generalized logarithmic mean bound for the Seiffert mean $P(a, b)$.

Proof. For any $0<\varepsilon<1$ and $x>0$, from (1) and (2), we have

$$
\begin{aligned}
& {[P(1+x, 1)]^{\varepsilon}-\left[L_{-\varepsilon}(1+x, 1)\right]^{\varepsilon}} \\
& \quad=\frac{f(x)}{\left[(1+x)^{1-\varepsilon}-1\right][4 \arctan \sqrt{1+x}-\pi]^{\varepsilon}},
\end{aligned}
$$

where $f(x)=\left[(1+x)^{1-\varepsilon}-1\right] x^{\varepsilon}-(1-\varepsilon)(4 \arctan \sqrt{1+x}-\pi)^{\varepsilon} x$. 
When $x \rightarrow 0$, then making use of the Taylor expansion, we get

$$
\begin{aligned}
f(x)= & {\left[(1-\varepsilon) x-\frac{1}{2} \varepsilon(1-\varepsilon) x^{2}\right.} \\
& \left.+\frac{1}{6} \varepsilon(1-\varepsilon)(1+\varepsilon) x^{3}+o\left(x^{3}\right)\right] x^{\varepsilon} \\
& -(1-\varepsilon)\left[x-\frac{1}{2} x^{2}+\frac{7}{24} x^{3}+o\left(x^{3}\right)\right]^{\varepsilon} x \\
= & \frac{1}{24} \varepsilon^{2}(1-\varepsilon) x^{3+\varepsilon}+o\left(x^{3+\varepsilon}\right) .
\end{aligned}
$$

Equations (48) and (49) imply that for any $0<\varepsilon<1$, there exists $\delta=\delta(\varepsilon)>0$ such that

$$
P(1+x, 1)>L_{-\varepsilon}(1+x, 1)
$$

for $x \in(0, \delta)$.

Therefore, Theorem 5 follows from inequalities (4) and (50).

\section{Acknowledgments}

This paper was supported by the Natural Science Foundation of China under Grant 61173123, the Natural Science Foundation of Zhejiang Province under Grants Z1110551 and LY12F02012, and the Natural Science Foundation of Huzhou City under Grant 2012YZ06.

\section{References}

[1] H. J. Seiffert, "Problem 887," Nieuw Archief voor Wiskunde (4), vol. 11, no. 2, p. 176, 1993.

[2] E. Neuman and J. Sándor, "On the Schwab-Borchardt mean," Mathematica Pannonica, vol. 14, no. 2, pp. 253-266, 2003.

[3] I. Costin and G. Toader, "Optimal evaluations of some Seifferttype means by power means," Applied Mathematics and Computation, vol. 219, no. 9, pp. 4745-4754, 2013.

[4] W. D. Jiang, "Some sharp inequalities involving reciprocals of the Seiffert and other means," Journal of Mathematical Inequalities, vol. 6, no. 4, pp. 593-599, 2012.

[5] W. M. Qian and B. Y. Long, "Sharp bounds by the generalized logarithmic mean for the geometric weighted mean of the geometric and harmonic means," Journal of Applied Mathematics, vol. 2012, Article ID 480689, 8 pages, 2012.

[6] H. N. Shi and S. H. Wu, "Refinement of an inequality for the generalized logarithmic mean," Chinese Quarterly Journal of Mathematics, vol. 23, no. 4, pp. 594-599, 2008.

[7] C. P. Chen, "The monotonicity of the ratio between generalized logarithmic means," Journal of Mathematical Analysis and Applications, vol. 345, no. 1, pp. 86-89, 2008.

[8] F. Qi, S. X. Chen, and C. P. Chen, "Monotonicity of ratio between the generalized logarithmic means," Mathematical Inequalities \& Applications, vol. 10, no. 3, pp. 559-564, 2007.

[9] G. Toader, "Seiffert type means," Nieuw Archief voor Wiskunde (4), vol. 17, no. 3, pp. 379-382, 1999.

[10] C. E. M. Pearce, J. Pečarić, and V. Šimić, “On weighted generalized logarithmic means," Houston Journal of Mathematics, vol. 24, no. 3, pp. 459-465, 1998.
[11] H. J. Seiffert, "Ungleichungen für einen bestimmten Mittelwert," Nieuw Archief voor Wiskunde (4), vol. 13, no. 2, pp. 195-198, 1995.

[12] C. E. M. Pearce and J. Pečarić, "Some theorems of Jensen type for generalized logarithmic means," Revue Roumaine de Mathématiques Pures et Appliquées, vol. 40, no. 9-10, pp. 789-795, 1995.

[13] K. B. Stolarsky, "The power and generalized logarithmic means," The American Mathematical Monthly, vol. 87, no. 7, pp. 545-548, 1980.

[14] J. Sándor, “On certain inequalities for means III," Archiv der Mathematik, vol. 76, no. 1, pp. 34-40, 2001.

[15] P. A. Hästö, "Optimal inequalities between Seiffert's mean and power means," Mathematical Inequalities \& Applications, vol. 7, no. 1, pp. 47-53, 2004.

[16] H. Liu and X. J. Meng, "The optimal convex combination bounds for Seiffert's mean," Journal of Inequalities and Applications, vol. 2011, Article ID 686834, 9 pages, 2011.

[17] S. Q. Gao, "Inequalities for the Seiffert's means in terms of the identric mean," Journal of Mathematical Sciences: Advances and Applications, vol. 10, no. 1-2, pp. 23-31, 2011.

[18] L. Matejíčka, "Proof of one optimal inequality for generalized logarithmic, arithmetic, and geometric means," Journal of Inequalities and Applications, vol. 2010, Article ID 902432, 5 pages, 2010.

[19] B. Y. Long and Y. M. Chu, "Optimal inequalities for generalized logarithmic, arithmetic, and geometric means," Journal of Inequalities and Applications, vol. 2010, Article ID 806825, 10 pages, 2010.

[20] Y. M. Chu, M. K. Wang, and G. D. Wang, "The optimal generalized logarithmic mean bounds for Seiffert's mean," Acta Mathematica Scientia B, vol. 32, no. 4, pp. 1619-1626, 2012.

[21] Y. L. Jiang, Y. M. Chu, and B. Y. Long, "An optimal double inequality between logarithmic and generalized logarithmic means," Journal of Applied Analysis. In press. 


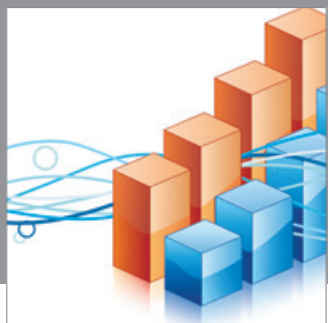

Advances in

Operations Research

mansans

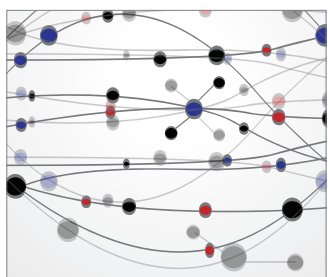

The Scientific World Journal
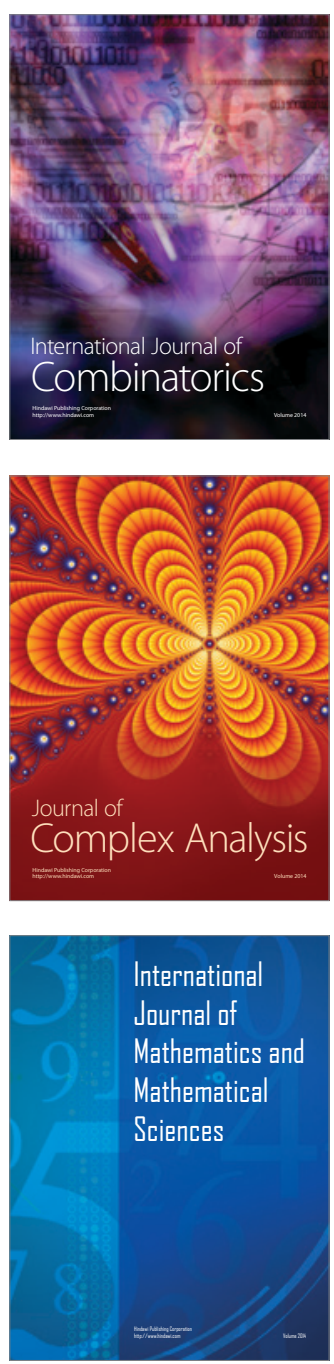
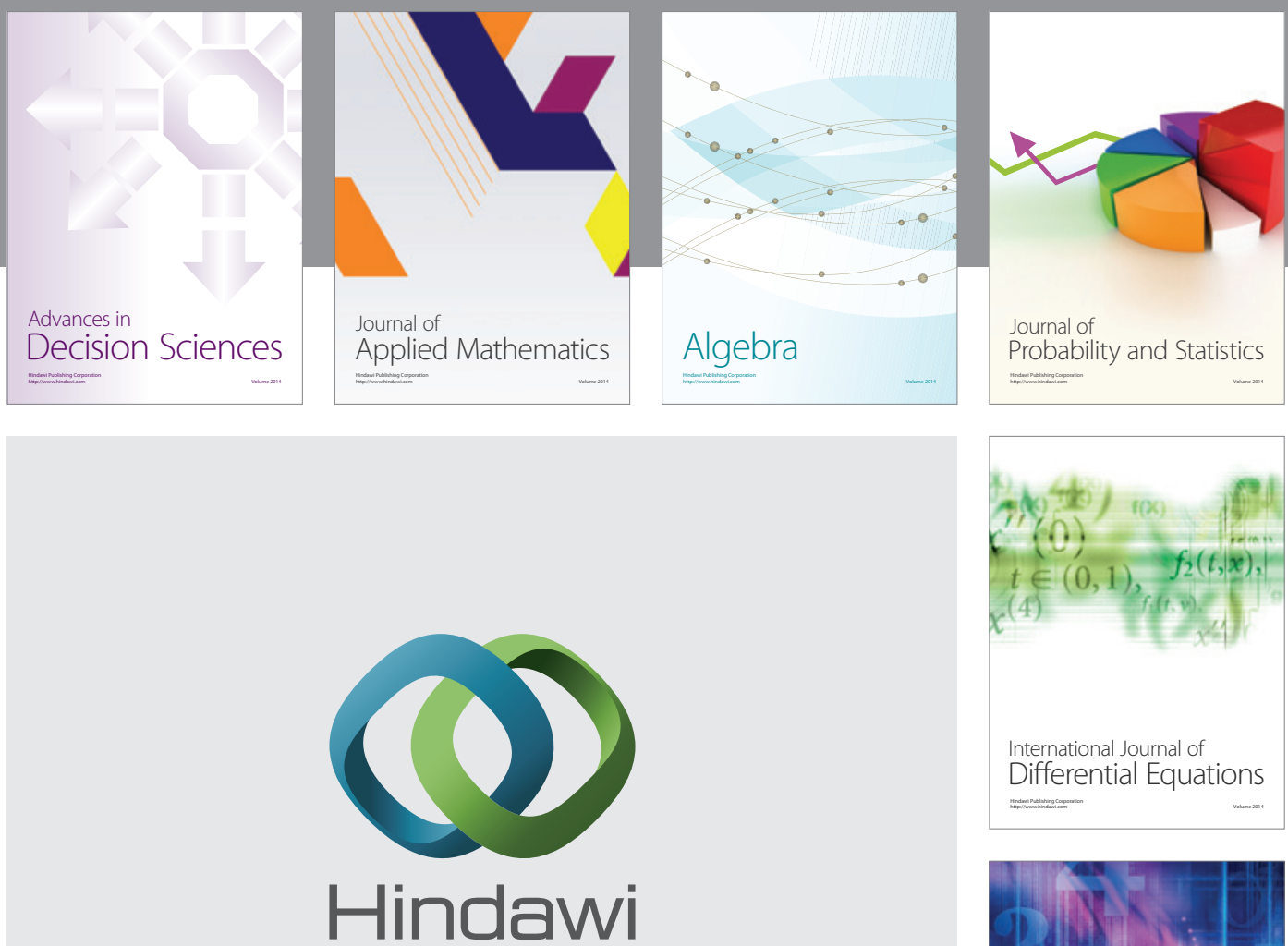

Submit your manuscripts at http://www.hindawi.com
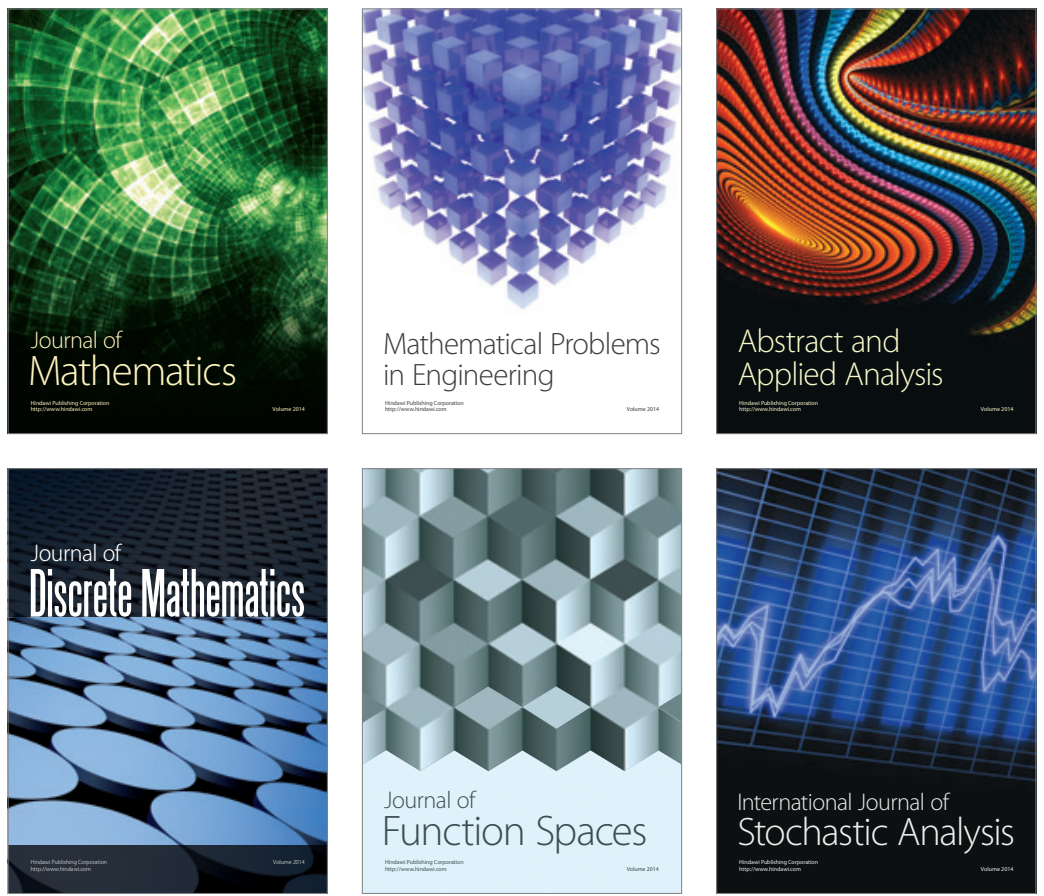

Journal of

Function Spaces

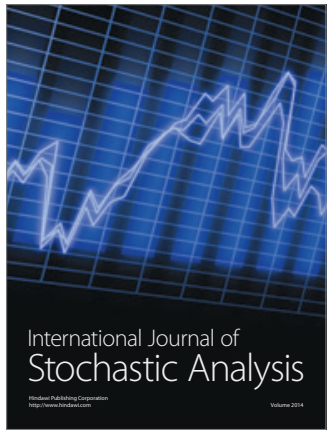

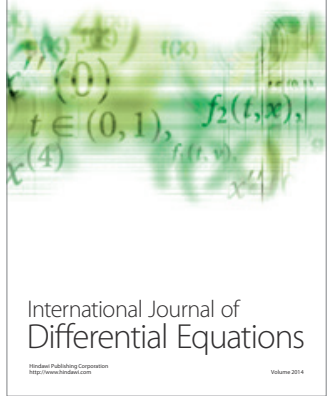
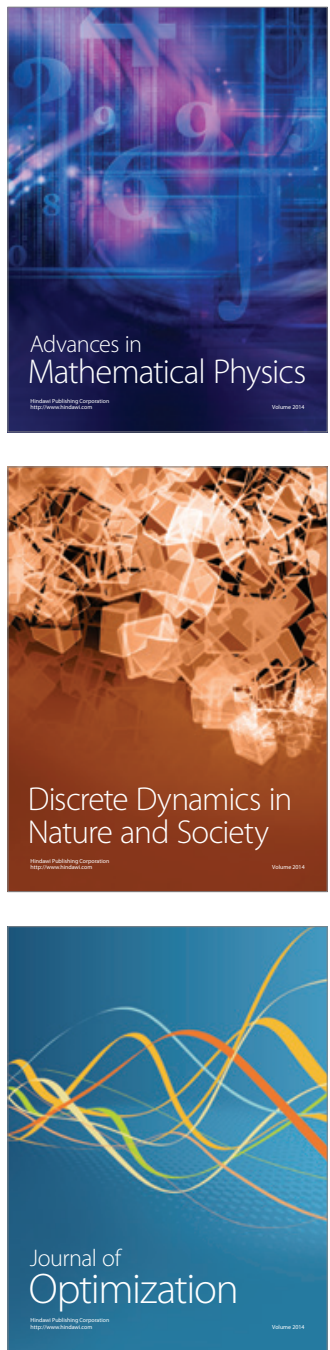